\title{
Through-thickness water transport and hygro-expansion in a paper sheet
}

\author{
Nik Dave $^{\dagger, \mathbb{P}, *}$, Ron Peerlings ${ }^{\dagger}$, Thierry Massart ${ }^{\mathbb{P}}$, and Marc Geers ${ }^{\dagger}$ \\ ${ }^{\dagger}$ Eindhoven University of Technology, $5600 \mathrm{MB}$, Eindhoven, The Netherlands \\ E-mail:n.dave@tue.nl,r.h.j.peerlings@tue.nl,m.g.d.geers@tue.nl \\ Web page: https://www.tue.nl/ \\ PUniversité Libre de Bruxelles, Avenue F.D. Roosevelt, 50 B-1050, Brussels, Belgium \\ E-mail: thmassar@ulb.ac.be \\ Web page: http://batir.ulb.ac.be \\ * Corresponding author: n.dave@tue.nl (Nik Dave)
}

\begin{abstract}
Paper, a hydrophilic material, is notably susceptible to deformations due to variations in moisture content, which develop over time. Understanding the moisture transport through the thickness of a paper sheet and the time-dependent mechanics allows us to study the curling behaviour of paper.
\end{abstract}

In this work, the time-dependent factors involved in deformation of a paper strip that is fully or partially wetted from one side and subjected to different boundary conditions is studied with a $1 \mathrm{D}$ numerical model. The different time-scales involved, in the process of imbibition in the inter-fibre pores and absorption (or water uptake) by the fibres, are analysed. The resulting hygro-expansion due to swelling of the wet fibres is then solved to predict the deformation and bending response of the paper strip.

We provide a phenomenological model here to describe the dynamic water flow through the thickness of a paper strip using the unsaturated flow theory. The numerical analysis shows a fair qualitative agreement with experimental observations.

\section{REFERENCES}

[1] Bosco, E., Peerlings, R.H.J., Lomans, B.A.G., van der Sman, C.G. and Geers, M.G.D., "On the role of moisture in triggering out-of-plane displacement in paper: From the network level to the macroscopic scale", International Journal of Solids and Structures (2017).

[2] Niskanen, K., Mechanics of paper products, Walter de Gruyter, (2012). 